\title{
Dichroism Infrared-reflective Thermochromic Ink of Mobile Terminals' Recognition
}

\author{
Baoning Sha and Shengyao Zhao \\ Shandong University, Institute of polymer research, Jinan, Shandong, China
}

\begin{abstract}
The decoration of thermochromic ink has applications ranging from engineering devices to liquid sensors. However, when it comes to accurate computer identification, the characteristic as dichroism is not fully used. Thus, we have recently proposed a manufacture method of dichroism infrared-reflective thermochromic ink. (DITI). By rubbing to heat DITI coated layer, this method enables us to finely and separately create dichroism manuscript after the handwriting is complete. Moreover, the microcapsules have cheerful compatibility. We have decorated conventional microcapsules with bola-type copolymer which allow us to fill them with Infrared (IR) reflective perssad. We consider this method promising to distinguish dichroism printing or writing on a tanglesome surface much quicker, for the IR reflective low-colour-density layer will be easily locked by an IR camera or a mobile phone camera to adjust specific characters of interest. We anticipate that this DITI ink will find use in markable temperature measurement, fluid machinery, anti-counterfeiting techniques and daily printing or writing.
\end{abstract}

\section{Introduction}

Thermochromic materials have significantly extended the applications of decoration, optical sensors and electronics. In recent years, thermochromic ink has been applied to various fields in life science [1,2] and engineering such as optical temperature measurement, fluid machinery, Anticounterfeiting techniques and daily printing or writing. DITI ink within flexible structures could open up new applications in electronics identification by adjusting the Chromogenic molecule with high-IR-reflective perssad.

Recent work has shown that temperature-sensitive ink and pressure-sensitive ink (PSI) can be used in fabricating a dual TSI and PSI array sensor by scientific grade inkjet printer, which showed a possible way in operating TSI[3,4]. However, a quick-direct distinguish of dichroism TSI on a tanglesome surface by a commercial camera still remains challenging. Applying the most conservative estimate to current demographic trends, the population of the ones who use mobile terminals will increase by about three billion people by the year 2050. Thus, a convenient way as distinguishing dichroism TSI could further broaden the usage among ordinary users[5,6]. Considering the complementary metal-oxide-semiconductor (CMOS) used in mobile terminals like smart phone has a wider sensitive wavelength in IR sections, we decide to plant several characteristics on traditional TSI.

Here we describe the design and demonstration of DITI system, where the heated IR reflective low-colourdensity layer can be precisely distinguished from the unheated layer, whereupon the quick-direct distinguish by mobile terminals is achievable. Distinct from previous reports, IR perssad is filled in the microcapsules mixed with low-colour-density parts so that DITI ink can be used in the similar way as ordinary ink and the distinguish of heated DITI layer can be driven through CMOS used in ordinary mobile terminals. The dichroism infrared-reflective thermochromic ink system concept involves (1) decorating conventional microcapsules with bola-type copolymer, (2) filling IR reflective perssad, (3) adjusting its typical wavelength in IR sections, (4) distinguishing different type of layers through commercial CMOS.

\section{Characteristics of DITI and bola- type copolymer}

\subsection{High fraction of coverage in high-colour- density layer}

Reversible thermochromic organic materials usually consist of at least three components: a colour former, colour developer and solvent[2,7]. Common colour formers are electron-donating, leuco dyes,. As these compounds change colour when $\mathrm{pH}$ is altered, they are in reality, halochromic. Frequently used examples include crystal violet lactone and thymolphthalein. Their reaction with the second component, the electron-accepting developer, defines the position of the longest wavelength absorption which causes colouration/decolouration $[3,8]$. For better understanding, we create a microcapsule 
simulation with low colour density, which is shown in figure 1.

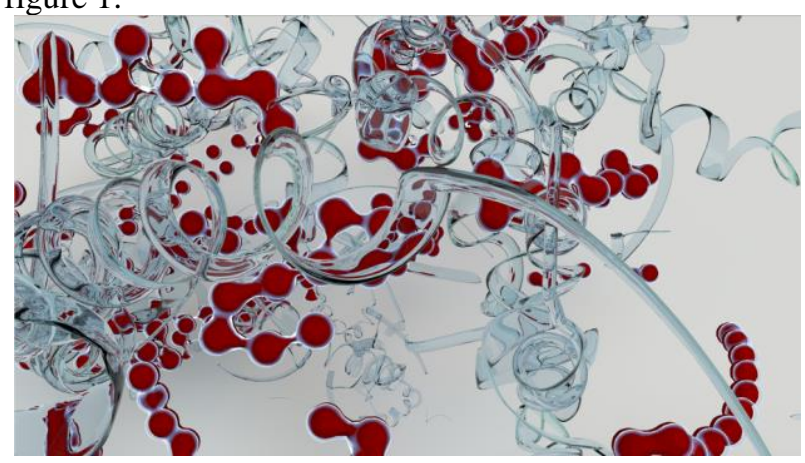

Figure 1. Model simulation made with Cinema 4D

The thermochromic pigment particles are analysed by using a Hitachi su70 field emission SEM. As only the particles locate at the very top of a dry screen-printed sample can be observed, more particles were made visible when the topmost layer of the binder was selectively etched by weakly ionised highly dissociated oxygen plasma. According to the previous study, plasma was created in a glass Pyrex tube with an inductively coupled RF generator operating at a frequency of 27,12 $\mathrm{MHz}$ and an output of $200 \mathrm{~W}$. The oxygen pressure was $75 \mathrm{~Pa}[9]$. The procedure is based on selective interaction of activated gaseous particles with the film coating. Selectivity of the etching process was achieved due to different oxidation probability of the polymer binder and TC pigments. The method is frequently used to detect distribution and orientation of particles in polymer matrix. The removal of the highest layers of polymer binder in UV-cured TC printing inks was completed in a couple of minutes. This way the top-laying TC pigment particles become visible on SEM micrographs.

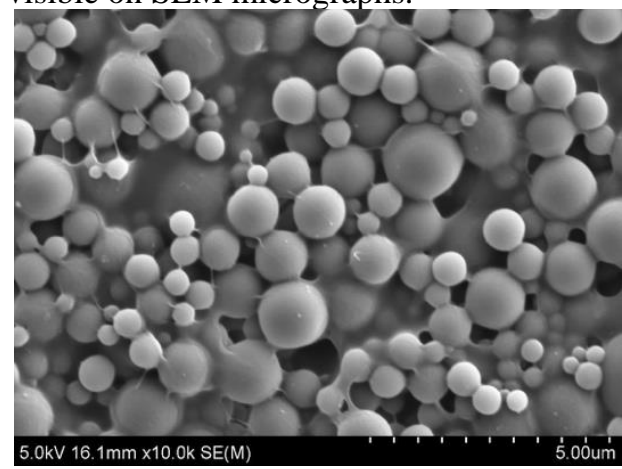

Figure 2. DITI painted layer (without reform)

Although the ink has similar pigment particle size distribution and comparably thick microcapsule shells with stability against oxygen plasma etching, we still need to develop a certain pattern that can protect the capsule from being oxidized, due to the unique electronaccepting developer decorated with IR perssad. The SEM of DITI painted layer (without reform) is schematically shown in figure 2.

Conventional pigments are relatively stable against activated neutral oxygen radicals which are responsible for selective etching in weakly ionised oxygen plasma.
The situation is different in systems with DITI ink: under some proportion of oil-type IR reflective perssad, DITI ink and additive can caused an aggregation. When they are destroyed, pigment particles change simultaneously so the method cannot be applied to evaluate the particle size distribution. However, this experiment shows that polymer envelopes are much more stable against selective etching than the binder itself; the binding energy of the molecular species in the polymer protecting capsule is much larger than it is inside the binder. Thus, a more efficient way to protect the binder, which contains with IR reflective perssad is the eye to see this system activated. We develop a prereaction by using DCPyn to protect the system from unwanted reactions with their surroundings. The SEM of DITI painted layer (with DCPyn) is schematically shown in figure 3 .

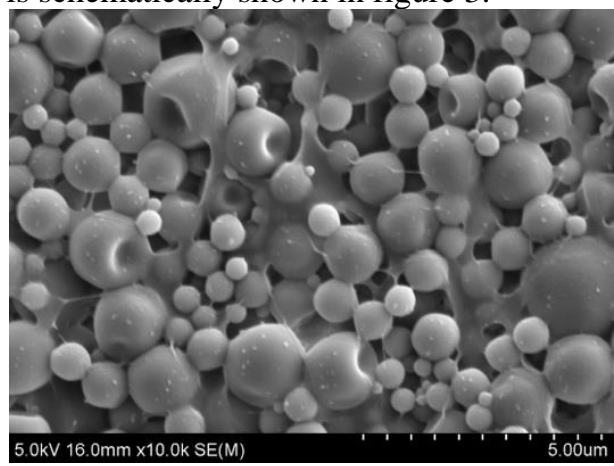

Figure 3. DITI painted layer (with DCPyn)

\subsection{Instant-high-temperature colour recovery}

Previous work had pointed out that the colourimetric properties of the reversible process were tested up to the temperatures at which a short exposure should cause no long-lasting colour change (up to $140^{\circ} \mathrm{C}$, according to the producer data $[10,11])$. As a condition of our handwriting or painted DITI to remain stable when being used, we measured several copolymers mixed with DITI in PreExperiment. The size of the largest particles in DITI inks was $12 \mu \mathrm{m}$; this is the so-called grindometer value. The DITI mixture was first added with DCPyn and thenpainted on PVDF membrane. The thickness of the dry layers was measured using a Talysurf (Taylor Talysurf i120). Those measurements finally led us to the found of new utilization in DCPyn, and they also brought us to an unrecorded phenomenon. We found that if the largest pigments could fill the entire thickness three times of dry layer (averagely $20 \mu \mathrm{m}$ ), the dry layer can recover from the low-colour-density states.

Reversible colour change occurs via two competing reactions namely that between dye and developer and the other between solvent and developer. The first of these interactions prevails at lower temperatures where the solvent exists in its solid form and gives rise to coloured dye developer complexes; at higher temperature the solvent melts, causing the solvent developer interaction to dominate[12-15]. Thus, dye developer complexes are destroyed which converts the system into its colourless state. Leuco dye developer solvent composites are by far the most important systems to achieve thermochromic properties using organic materials. The temperature at 
which decolour- isation/colourisation occurs is controlled by the melting temperature of the solvent. We think the Instant-high-temperature colour recovery may be caused by the catalytic system of DCPyn, electron-donating, electron-accepting and PVDF membrane. The longest heating time of the accurate of colour recovery phenomenon is related to the proportion of DCPyn. It also confirmed that large proportion of DCPyn (about $4.5 \%$ )will Reduce the number of decolourisation/colourisation reaction cycle.

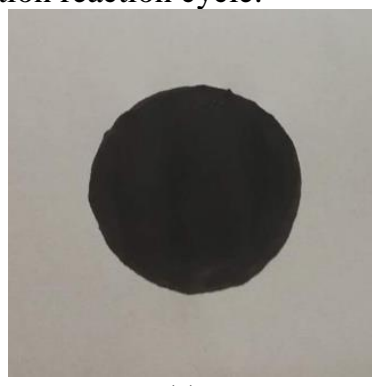

(a)

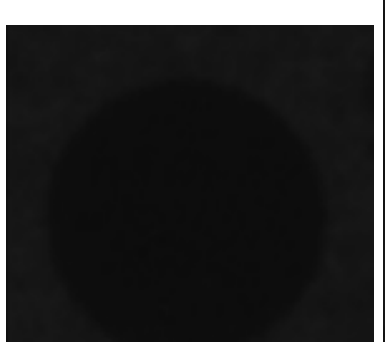

(c)

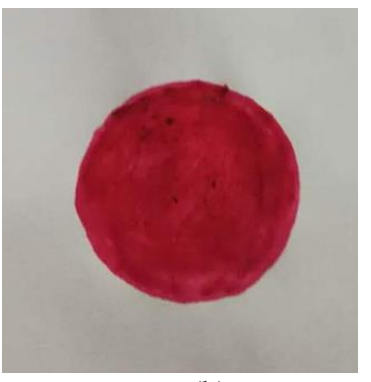

(b)

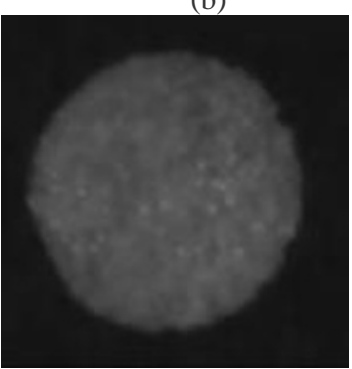

(d)
Figure 4. Images of (a) unheated and (b) heated layers captured with SLR camera. Gray scale images captured with band-pass filter of $530 \pm 60 \mathrm{~nm}$ when the layer is (c)unheated or (d)heated.

\section{Properties of written DITI layer}

\subsection{Sensitivity}

Here, the final expected application is mostly used by ordinary mobile terminals. We used RGB colour models to measure the sensitivities of different types of DITI. The emission images were captured by several mobile terminals that contain different type of CMOS and a commercial SLR camera (Canon 760D).

To get the data of its typical wavelength in IR sections and test the stabilization of the scanning recognition system, we attached Infrared filters to the lens of the commercial SLR camera (Canon 760D). As shown in figure 4 the emission images can be separately detected by the Infrared filters. We confirmed that the isolated ink mark of the two states (colourless and coloured) can be recognized by mobile terminals and can be recognized even better if the incident spectrum contains infrared.

\section{Conclusion}

We have proposed a DITI system. The heated lowcolour-density layer can be finely distinguished and adjusted by the scanning (CMOS) system we built. By carefully discussing the setting of ink components, the thermochromic microcapsules is peacefuly stacked on the surface of DITI layer, leading to prevent the unheated DITI from being written or printed at a low-colourdensity rank. The characteristics of DITI on IR reflection is a promise of commercial distinguish. Special Emission spectra in IR sections is another key to accomplish the quick-direct distinguish by mobile terminals. We also endued DITI with some tricky characteristics by adding bola-type copolymer(DCPyn), such as colour restoration, high-colour-density structure and isolation from being affected by the IR perssad. These characteristics mentioned above perfectly match the needs in electronics identification and high functioning paper work, which could also be a promise of sustainable connection between electronic office and traditional office.

\section{Acknowledgments}

This work was financially supported by Carbon Fiber Engineering Research Center of Shandong Province in China, and by the integration projects of Shandong University. Both are gratefully acknowledged.

\section{References}

1. J W Gregory, Porous pressure-sensitive paint for measurement of unsteady pressures in turbomachinery 2004, AIAA Pap. 0294.

2. J W Gregory, K J Disotell, D Peng, T J Juliano, J Crafton and $\mathrm{N}$ M Komerath, Inverse methods for deblurring pressure-sensitive paint images of rotating Surfaces 2014, AIAA J. 52 2045-2061.

3. Maclaren DC and White MA. Dyeedeveloper interactions in the crystal violet lactone-lauryl gallate binary system: implications for thermochromism. Journal of Materials Chemistry $2003131695 \mathrm{e} 700$.

4. Maclaren DC and White MA. Competition between dyeedeveloper and sol-ventedeveloper interactions in a reversible thermochromic system. Journal of Materials Chemistry $2003131701 \mathrm{e} 4$.

5. Zhu CF and Wu AB. Studies on the synthesis and thermochromic properties of crystal violet lactone and its reversible thermochromic complexes. Thermochimica Acta 2005425 7e12.

6. Maclaren DC and White MA. Design rules for reversible thermochromic mixtures. Journal of Materials Chemistry $200540669 \mathrm{e} 76$.

7. Seeboth A, Klukowska A, Ruhmann R and Lötzch D. Thermochromic polymer materials. Chinese Journal of Polymer Science 200725 123e35.

8. White MA. Thermochromism in commercial products. Journal of Chemical Education 199976 $1201 \mathrm{e} 5$.

9. Mozetic M. Controlled oxidation of organic compounds in oxygen plasma. Vacuum 200371 $237 \mathrm{e} 40$.

10. Vesel A, Mozetic M, Hladnik A, Dolenc J, Zule J and Milosevic S. Modification of ink-jet paper by oxygen plasma treatment. Journal of Physics DApplied Physics 200740 3689e96. 
11. Kunaver M, Klanjsek Gunde M, Mozetic M and Hrovat A. The degree of dispersion of pigments in powder coatings. Dyes and Pigments 200357 $235 \mathrm{e} 43$.

12. Y. Matsuda, T. Uchida, S. Suzuki, R. Misaki, H. Yamaguchi and T. Niimi. Pressure-sensitive molecular film for investigation of micro gas flows. Microfluid Nanofluid. 2011 165-171.
13. Ma C, Xia Y. Colloids Surf 199266215.

14. Ma Z., Chen M and Glass J E. Colloids Surf 1996 112.

15. Y. Egami, K. Fujii, T. Takagi, Y. Matsuda, H. Yamaguchi and T. Niimi. Reduction of temperature effects in pressure-sensitive paint measurements. AIAA J. 512013 1779-1783. 